high, lights of our editorial policy. For the most part these series of articles will be invited directly by the Editor, but manuscripts of this type will gladly be accepted at the Editor's discretion.

3. Practical, teaching and historical articles.-The popular series, known as "Practicalities," will be revived, and again every possible branch of medicine, surgery, etc., will be covered in due course.

4. Clinical case reports.- Reports of any interesting case or series of cases illustrating special problems of diagnosis or treatment are urgently invited. Every report may be illustrated by radiographs, pathological investigations, etc.

5. Book page.-This new feature will include a list of all new publications, with titles, précis, and publishers' names, together with commentaries and reviews.

6. Drug page.-This new feature will include a list of all new drugs placed on the market by reputable firms, and the results of clinical trials of any new drug will be invited and published.

7. Post-graduate teaching and medical news bulletin.-This new feature will be a programme of great interest to many of our readers, and will include notes from all the large provincial and metropolitan centres. It will be put in as a central inset which can be torn out and placed in any convenient pocket or drawer, so facilitating ease of reference.

Gradually further sections and items of topical interest will be added.

In all cases articles will be profusely illustrated whenever possible by coloured plates, photographs, radiographs, microphotographs, line drawings, etc. Such illustrations are not only helpful to the author in his description but also to the reader in his understanding thereof.

We believe that this new and bold editorial policy will bring greater interest to our readers. And let it be noted that all this will be done without increasing the cost of the journal. May we ask for your continued support and for your help by personal recommendation to your professional friends who do not subscribe to the journal? We trust that our twenty-first year of publication will prove to be a momentous one for all concerned.

\title{
THE ANATOMY AND PHYSIOLOGY OF MICTURITION AND THE EFFECT OF VARIOUS NEUROLOGICAL DISORDERS UPON THIS FUNCTION
}

\author{
By I. ANSELL, M.B., M.R.C.P. \\ (Resident Medical Officer, Liverpool Royal Infirmary; Demonstrator in Medicine, \\ University of Liverpool)
}

The bladder is a hollow, muscular organ, having an ovoid shape when distended, folding up to a flattened tetrahedron when empty. It is situated in the anterior part of the pelvis, with its apex pointing forwards towards the symphysis pubis, and then continued upwards as the urachus, a fibrous cord leading to the umbilicus.

The superior surface is slightly dome-shaped, and is covered by the peritoneum of the pelvic floor, coming into relationship with coils of small intestine and pelvic colon, and sometimes the appendix, also the uterus and appendages in the female. This surface is continuous posteriorly with a triangular-shaped base-the trigone, which is separated from the rectum by the fascia surrounding the vasa deferentia and seminal vesicles in the male, and the fornices of the vagina enclosing the cervix uteri in the female.

The trigone has two superior angles which are pierced obliquely by the ureters coming down from the kidneys. Its inferior angle is continuous with the funnel-shaped neck of the bladder, which rests on top of the prostate, and leads to the urethra through the internal urethral orifice guarded by the internal sphincter. 
In the female, the neck of the bladder is in relationship to the anterior wall of the vagina, continuous with a short urethra of one and a half inches length, which is closely attached to this wall by strong fibrous tissue.

Laterally, the bladder is bounded in its anterior part by the obturator internus, and posteriorly it is separated from the levator ani by a plexus of blood vessels supplied by the superior and inferior vesical arteries and veins, branches of the internal iliac vessels. These are accompanied by lymphatics which drain into the internal iliac and sacral lymph glands.

In front, the apex lies behind symphysis pubis when empty, but as the bladder fills the apex rises out of the pelvis, separating the peritoneum from the posterior surface of the anterior abdominal wall. Below it is continuous with the urethra, which in the male traverses the prostate for a distance of one and a quarter inches, and the external sphincter of the membranous part (compressor urethrae muscle), leading into the bulbous and penile urethra to the external urinary meatus.

The inner surface of the bladder presents a rugose appearance, becoming smooth when distended. The trigone always remains smooth, and the ureteric orifices at its superior angles are separated by the inter-ureteric bar of muscle. The lining which consists of a mucous membrane of transitional epitheleum loosely attached to the underlying muscular coat, has a cream colour when examined by the cystoscope, this becoming pinker especially towards and over the trigone.

The muscular coat consists of three layers of smooth muscle, arranged in an outer longitudinal layer continuous with the muscle of the prostate and attached in front to the pubis; a middle circular layer with its modified fibres extending from the ureteric orifices down to include the internal urethral opening as the internal sphincter. The thin, inner layer has an oblique distribution, and is incomplete.

The bladder receives its nerve supply through the nervi erigentes which carry the parasympathetic fibres from the nuclei in the lateral horns of the second, third and fourth sacral segments of the cord. These fibres pass through the hypogastric ganglia without relaying, to end in the local ganglia of the detrusor and circular muscle of the bladder. These nerves also carry the sensory fibres from the bladder wall, subserving the sensation of touch, pain and pressure back to the cord, via the posterior nerve roots and the postero-lateral columns, to the post-Rolandic area of the cerebral cortex.

Another source of supply is from the hypogastric nerves which descend from the hypogastric plexus, carrying the preganglionic sympathetic fibres from the first and second lumbar segments of the cord, to relay in the hypogastric ganglia behind the bladder, termirfating in the circular muscle, especially the part forming the internal sphincter, and also in the detrusor muscle. The hypogastric nerve also carries the pain sensation of spasmodic contraction from the bladder up to at least the ninth thoracic segment of the cord, via the'posterior nerve roots. Another nerve which takes part in the control of micturition is the pudic nerve arising from the first, second, third and fourth sacral segments, and supplying the external sphincter; which is under voluntary control as far as closure is concerned.

We have thus a visceral reflex arc with its centre in the lumbo-sacral cord, under the influence of the higher centres, by virtue of the upper motor neurone, the mechanism of the normal reflex being as follows:-

The urine is excreted into the renal pelves and peristaltic contractions passing along the ureters at the rate of three a minute drive the urine down to the bladder, entering through the oblique valvular orifices in the trigone.

As the urine accumulates, the bladder wall is gradually distended until a volume of approximately 200 c.c.s sets up rhythmical waves of contraction, under the restraining control of the local centre in the sacral cord. This process is subconscious and does not intrude upon consciousness until a volume of 400 c.c.s at about fifteen to eighteen cms. of water pressure causes the vesical contractions to reach a threshold intensity. The impulses from the distended wall, due to increase of tension, travel via the parasympathetic fibres of the nervi erigentes to the posterior nerve roots of the second and third sacral segments, and ascend the postero-lateral columns of the cord to the sensory cortex in the post-Rolandic area. Here the sensation is appreciated as a vague discomfort in the anterior part of the perineum, radiating along the urethra at irregular intervals. If the bladder is greatly distended, then somatic pain due to irritation of nerves in surrounding structures causes localised pain, particularly in the hypogastrium, but sometimes referred down the front of the thigh in its upper two-thirds. 
If convenient, the motor cortex sends excitory stimuli down the lateral columns to the lumbo-sacral centre, intensifying the detrusor contractions at the same time, causing efferent impulses to travel along the nervi erigentes, and thus reciprocally relaxing the external sphincter, relaxed via the pudic nerve as part of a general perineal muscular relaxation.

This allows a few drops of urine to trickle into the prostatic urethra, intensifying the reflex contraction of the bladder wall. Helped by increasing intra-abdominal pressure due to contraction of the abdominal wall and fixation of the diaphragm, the bladder is emptied, the last few drops being squeezed out of the posterior urethra by the contractions of the sphincter muscle and bulbo-cavernosus muscle as the perineal musculature takes up its original tone.

When conditions are not convenient for emptying the bladder, inhibitory impulses are received from the pre-Rolandic area by the sacral centre, and the vesical contractions are inhibited. At the same time, the internal sphincter is tightly closed by impulses, travelling in the sympathetic fibres of the hypogastric nerves, which also have an inhibiting effect on the detrusor muscle, allowing it to relax, and the bladder to accommodate more urine. Further distention sets up more waves of contraction which become more difficult to inhibit, and require the assistance of the voluntary external sphincter and perineal musculature with adduction of the thigh muscles. When the pressure reaches roo cms. of water, the pain is unbearable, and the urine forces itself past the sphincters, dribbling down the urethra. At this stage, there is no voluntary control, and the bladder begins to empty, slowly at first, until the effects of inhibition on the stretched musculature wears off. Later, the vesical contractions reappear, and the rate of urinary flow increases. After a certain degree of emptying has taken place, the flow can be cut short by voluntary contraction of the external sphincter, and inhibitory impulses from the higher centres causing the detrusor muscle to relax. As the bladder empties the hypogastric pain diminishes, becoming a vague discomfort, and persisting faintly into consciousness for several minutes after emptying.

An interesting corollary which follows as a result of the great distention is the presence of temporary frequency, no doubt due to the sudden diminution of intravesical pressure causing the urine in the ureters and renal pelves to flow in quickly. Volumes as small as 50 c.c.s cang initiate micturition when conscious effort combined with perineal relaxation is attempted, but increased intra-abdominal pressure in itself has no effect in initiating micturition, and cannot relax the external sphincter.

The perfection of this series of conditioned reflexes in the child requires time and education. The more primitive reflex evacuation with smaller volume which occurs at frequent intervals is conditioned to the normal evacuation at appropriate times. During this process, should the higher control be diminished, as for example during sleep, the bladder may revert to its primitive irritability and empty involuntarily. At the other extreme, the bladder can be trained to accommodate larger volumes without discomfort, this being more common in the case of women and sedentary workers. Similarly "bladder consciousness" (the subject thinking of micturition) may produce vesical contractions, and result in a willed evacuation of a far smaller volume of urine than would normally be required to produce adequate stimulation.

\section{Effects of Neurological Disorders on Bladder Function.}

For the normal act of micturition to occur, it is essential that the reflex arc and its connections should be intact. Interference with this arc causes impaired function, the nature of which depends upon the site of the lesion and the rate at which it is produced. Such lesions can be congenital, traumatic, inflammatory, vascular, degenerative, toxic, or neoplastic.

Neurological disorders of the bladder will be classified here according to the site of the main lesion, and the resulting symptoms and signs will be described for some of the commoner pathological processes.

A. Lesions of the Reflex Arc.

I. Afferent neurone:-

Parasympathetic sensory fibres in the posterior nerve roots.

2. Afferent and efferent neurones:-

Sympathetic sensory and motor fibres in hypogastric and presacral nerves.

Parasympathetic sensory and motor fibres in nerve roots and nervi erigentes.

3. Anterior horn cells in sacral cord.

4. Reflex centre in lumbo-sacral cord. 
B. Lesions of the Upper Motor and Sensory Neurones.

I. Motor and sensory fibres in the spinal cord.

2. Motor fibres in cortico-spinal system and cortex.

3. Cerebellum.

4. Thalamus.

5. Extra-pyramidal.

C. Psyche.

\section{A. I. Lesions of the Afferent Neurone.}

In tabes dorsalis the site of the lesion is in the posterior nerve roots of the lumbo-sacral segments, thus interfering with the proprioceptive sensory impulses which subserve the tonic reflexes. This leads to a loss of tone of the bladder wall, with difficulty in starting the act, sluggishness of urinary flow, and terminal dribbling. Sometimes acute retention is the first symptom.

At the same time, the sensations which inform the patient that his bladder is full, are diminished, and the desire to micturate enters consciousness infrequently.

As the degeneration becomes more extensive, sensation may be completely lost, and urine accumulates in the distended bladder, which now takes on an automatic function by means of the intact local vesical plexus in the bladder wall. The resulting contractions are weaker than normal, and do not empty the bladder completely, leaving a residual content which may increase to as much as 300 c.c.s. When the volume of residual urine reaches the bladder capacity, a form of overflow incontinence occurs and the patient complains of frequency.

Should the residual urine become infected, then the toxins produced are absorbed, and depress the normal function of the local vesical nerve plexus and muscle contractions. This leads to complete atonicity of the bladder wall, which now acts like an inert elastic bag. When the contents reach volumes of 800 c.c.s or more, the resistance of the sphincter is overcome and true overflow incontinence results in continuous dribbling. If the infection is cleared up, the tone usually returns and the bladder becomes automatic again. Failure to overcome the infection may result in ascending pyelonephritis and death from uraemia. If the bladder wall is healthy, the motor side of the arc still functions, and the patient can micturate whenever he wishes.

The diagnosis of this neurogenic bladder should be made by routine neurological examinations, and can be confirmed by cystoscopy when a fine trabeculation is seen, and by cystometry.

These facts are useful indicators of the line of treatment to be followed in early cases. If the patient attempts to empty his bladder at regular and frequent intervals, the residual urine is kept down to a minimum and over-stretching prevented. Advanced cases with infection should have a high suprapubic catheterisation with tidal drainage, rather than the frequent passage of urethral catheters or indwelling catheters with their resultant urethritis.

In the early stages of tabes, stimulation of the posterior nerve roots may occur, producing vesical crises, in which paroxysms of lancinating pain are referred to the tip of the penis.

The sensory fibres are sometimes involved in spina bifida causing a similar type of bladder syndrome to tabes.

\section{A. 2. Afferent and Efferent Neurones.}

(a) Lesions of the sympathetic fibres in the hypogastric and presacral nerves interfere with motor as well as afferent impulses. An example is provided by sympathectomy, which is sometimes carried out for the relief of pain in diseases of the uterus and bladder. This results in relaxation of the internal sphincter and trigone, with dilatation of the ureteric orifices. The effect on the internal sphincter produces dribbling, incontinence, and frequency, which soon passes off, usually within three weeks. Micturition is otherwise undisturbed. The relief of pain is not always complete, and may require further measures, such as section of the sacral parasympathetic nerves.

(b) Lesions of the parasympathetic (sensory and motor fibres) may accur after operations for the excision of the rectum or other pelvic organs; lesions of the cauda equina due to injury, inflammation or neoplasm; spina bifida, or after spinal anaesthesia. These produce what is known as the "isolated bladder." 
In the case of a sudden lesion, a period of shock causes retention. Later, because of inter-a ruption of efferent stimuli, voluntary micturition cannot take place, and if automatic emptying ${ }_{z}$ occurs the vesical contractions are poor.

These contractions depend upon the local neuromuscular reflexes, and helped by increasing ${ }^{\circ}$ intra-abdominal pressure, result in partial expulsion of the bladder contents, leaving a large: residuum of 300 c.c.s. Cystogram shows the "cottage-loaf" outline and funnel-shaped neck.

Pain from over-distention is appreciated if the sympathetic is intact, as this enters theo cord as high as the ninth dorsal segment. In retention due to partial lesions of the parasympathetic, sympathectomy may be helpful (Learmouth). bladder.

Polyneuritis of all forms may also affect the sacral nerves and similarly produce an atonice

A. 3. The Anterior Horn Cells in the sacral cord may be affected by anterior poliomyelitis. 0 This may cause atrophy of the external sphincter. Sometimes there is transitory involvementof the autonomic which usually recovers within seven days, but sometimes only partially.

A similar syndrome occurs in certain lesions of the cauda equina when faecal as well as urinary incontinence results. This commonly followed the administration of intrathecal injections of soluble sulphapyridine in the early days, before its irritant effect upon the meninges: was recognised.

\section{A. 4. Reflex centre in lumbo-sacral cord.}

Lesions of the reflex centre itself occur in injuries of the conus medullaris, and are similarew to those of the parasympathetic, except that the normal sensations of distention may be pre-o served.

\section{B. I. Lesions of the Upper Motor and Sensory Neurones in the Spinal Cord.}

Sudden injuries causing complete transection of the cord may result from fractures, dislo- $\frac{\mathbb{D}}{3}$ cations, or bullet wounds of the spine, which may be associated with contusion, compressiof or concussion. Any of these three conditions can occur individually, but in their initial stages give rise to a similar picture.

"Spinal shock" is the initial symptom, however high or low the lesion may be situated.

'The detrusor muscle becomes flaccid, but the tone of the internal sphincter soon returis, resulting in complete retention with loss of voluntary control. The bladder now acts like an elastic bag, the accumulating urine distends and overstretches its fibres. When the intravesicap pressure is high enough to overcome the internal and external sphincters, urine escapes ando retention with overflow results. Unless the retention is relieved, infection usually follows.

If the patient avoids infection, in seven to twenty-one days when the shock and contusion have worn off, tone may return in the detrusor muscle, and the vesical distention sets up a을 summation of contractions through the local reflex in the unimpaired cord, so that the bladder is incompletely evacuated at irregular intervals. This "automatic bladder" is not under volun-宩 tary control, nor does the patient know when he is about to micturate.

The bladder may empty with a content of 380 c.c.s under these conditions, but shouldo the patient increase his intra-abdominal pressure by coughing, straining, or even breathing:deeply, the evacuation can occur with smaller volumes such as 80 c.c.s. Similarly the induction of the flexion reflex, catheterisation, stimulation of the glans penis or any area supplied by the sacral nerves, may cause the automatic bladder to expel its contents.

With mild chronic infection the neuro-muscular irritability is increased, and voiding is frequent with small volumes and complete emptying.

In practice it is unwise to await the advent of automatic micturition, and if nothing happens within twenty-four to forty-eight hours, then a high suprapubic catheterisation with tidal drainage should be performed to prevent the onset of infection. This also brings on the automatic state earlier.

Gradually progressing lesions of the cord due to syphilis, inflammation, or tumours usually manifest themselves by difficulty in starting micturition, which might even occur without the patient's knowledge. The automatic stage is later established, and is associated with a residuale urine of 250 to 400 c.c.s.

If this does not occur, then retention with overflow indicates the necessity for a permanent㷒 suprapubic catheterisation. 


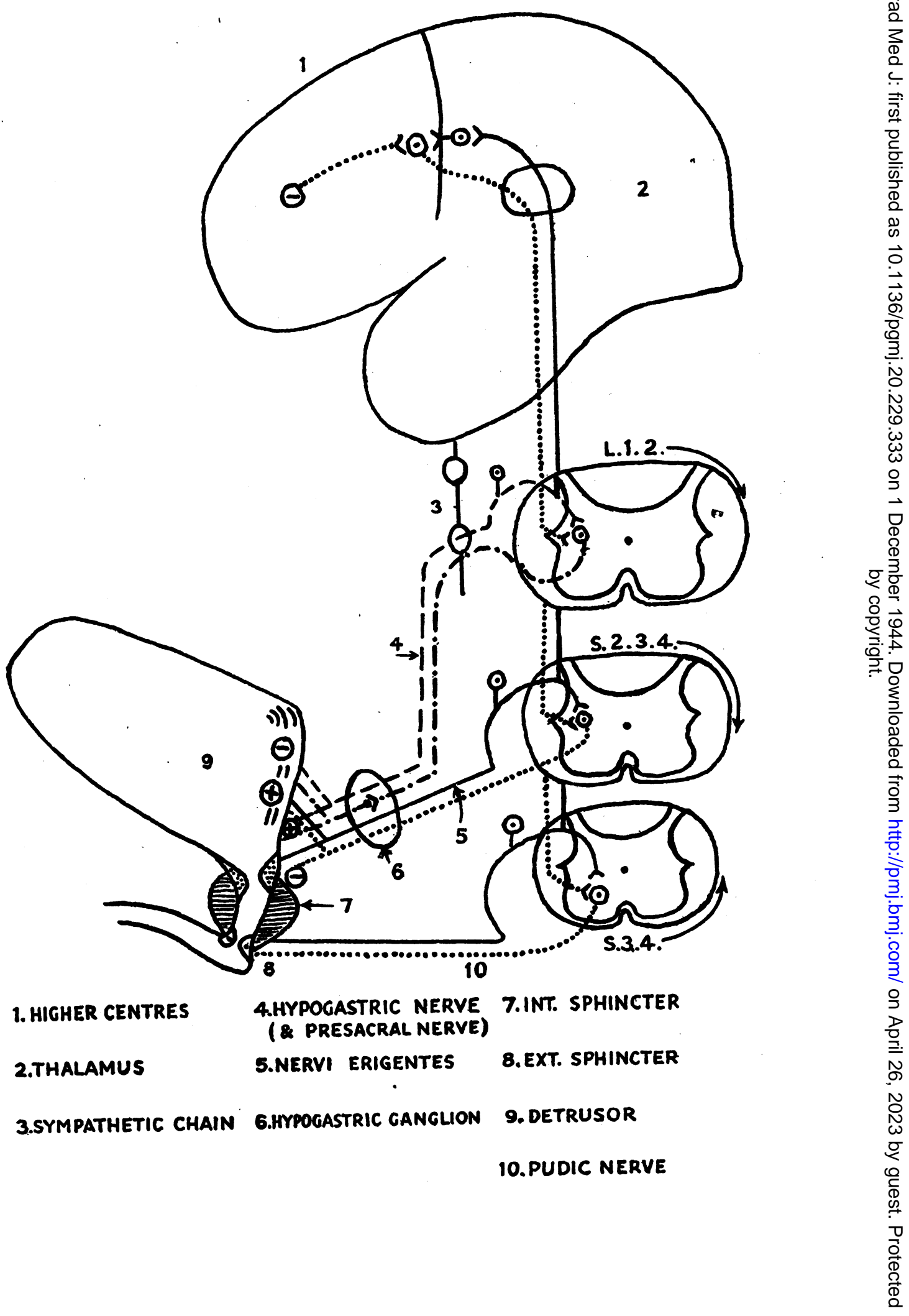


Internal sphincteromoty is sometimes successful when combined with drugs which cause detrusor contraction, such as acetyl, beta, methyl, choline, and chloride (the proprietary preparation being Mecholyl, in tablets of $0 . \mathrm{I}$ gm.), or Carbamylcholine Chloride (Carbachol, B.P., Moryl or Doryl, in tablets of two to four mgms.). These tablets can be taken by mouth at regular intervals, such as every four hours during the day, or after meals. More often than not the response to the drug wears off, and atonicity and secondary infection supervenes.

\section{B. 2. Lesions of the Motor Fibres in the Cortico-Spinal System.}

The onset may be:-

(a) Acute-as occurs in trauma, disseminated sclerosis, and vascular lesions. Cerebrospinal shock at first causes retention. After this has worn off, there is difficulty in starting micturition (loss of function), and the patient experiences urgency and frequency (release phenomena).

(b) Gradual-such as syphilis, disseminated sclerosis, postero-lateral sclerosis, subacute combined degeneration, amyotrophic lateral sclerosis, syringomyelia and neoplasm, producing increased urgency and frequency, followed later by incontinence.

Lesions of the cortex-e.g. cerebral tumours, may affect mainly the motor, sensory or psychic areas, according to the site of the growth. Difficulty in starting micturition follows when the motor area is involved, retention in the case of the sensory area, and incontinence in the psychic areas such as the frontal.

B. 3. Acute Cerebellar Lesions-e.g. injury, or posterior-inferior cerebellar artery thrombosis, gives rise to atonicity of the bladder wall, consequently there is hesitancy, retention, and an increased capacity with diminished expulsive power, resulting in a larger volume of residual urine.

This brings to mind the history of a middle-aged man who was admitted to a small hospital in a semi-conscious state, with acute retention and vomiting. He fell into the hands of a keen surgeon, who made a diagnosis of uraemia and discovered slight enlargement of his prostate. The prostate was removed and the man recovered, but left the hospital with a right hemiparesis, unsteadiness of gait, and numbness of the left side of his face. On examination later, he ex hibited signs of a recent left inferior cerebellar artery thrombosis which accounted for his retention of urine and vomiting, the latter having produced an extra renal uraemia.

B. 4. Lesions of the Thalamus may include such symptoms as vesical tenesmus and frequency in the syndrome (Dezerine and Egger).

B. 5. Lesions of the Extra-Pyramidal System include the corpus striatum or any of its connections with the substantia nigra, red nucleus, and subthalmic nucleus. These can be affected by infection, toxaemia, or degeneration, etc., with the production of the Parkinsonian syndrome. This consists of spasticity, tremor, and weakness of the somatic muscles, the vesical muscle acting similarly, showing high resting and emptying pressure of 20 to $40 \mathrm{cms}$. of water with cystometry.

Frequent and complete emptying occurs under the control of the will, and this is helped by hyoscine hydrobromide or other atropine-containing drugs used in the treatment of the syndrome.

\section{Functional Lesions (Psyche).}

Hysteria may produce retention, fear may initiate the condition by sympathetic inhibition of micturition, and later the mental dissociation isolates the vesical sensations from consciousness.

In contrast, anxiety and emotion increase the muscular tension of the bladder wall, setting up the normal reflex at frequent internals with smaller volumes.

It is suggested that enuresis, in which organic disease has been excluded, has a similar basis, psychological factors, together with faulty training being important.

Backus and Mansell (British Medical Journal, October I944) have recently shown that certain personality types are common in this disorder-the "timid, immature, dependent, often frus- ㅇ trated type" in $48 \cdot$ or per cent of adult patients, the average personality occurring in 27.07 per cent. Cystometry showed small bladders in 25 per cent of this series.

\section{Summary}

The anatomy and physiology of micturition is described.

The effect of lesions at various anatomical sites on normal function is discussed with reference to common pathological processes causing disease. 